Rechnungswesen

\section{Bremst die Doppik Investitionen?}

Ist die kamerale Buchführung für öffentliche Haushalte besser als die doppelte Buchführung (Doppik), weil dort mehr investiert wird? Die Frage nach dem Nutzen der Doppik ist zweifellos auch heute noch berechtigt und hätte bei der Einführung Gegenstand einer Gesetzesfolgenabschätzung sein sollen. Aber die Frage des Umfangs der Investitionstätigkeit hätte dabei sicher nicht im Vordergrund gestanden.

Grundlage für die Einführung der Doppik war das Ressourcenverbrauchskonzept. Darin geht es darum, den jährlichen Werteverzehr der mit Investitionen geschaffenen Anlagen und Einrichtungen zu dokumentieren. Damit sollte der kameralistischen Illusion, die Nutzung sei "irgendwie“ kostenlos, entgegengewirkt werden. Ganz neu war der Gedanke nicht, denn auch im kameralen Haushaltsrecht war eine Folgekosten- oder Folgelastenbetrachtung vorgesehen. Doch das ist keineswegs Standard in kommunalen Haushalten gewesen. Die Annahme, die Doppik hätte dazu führen sollen, dass mehr investiert werde, weil nun die geschaffenen Anlagen auf der Aktivseite der Bilanz erscheinen, ist merkwürdig. Denn eine als öffentliche Investition klassifizierte Maßnahme ist nicht per se besser als öffentlicher Konsum, wie die Debatte um Bildungsausgaben stets vor Augen führt.

Nun ist die Doppik längst aus dem Stadium des Pilotprojekts heraus, das man auch wieder beenden könnte. Die Doppik ist in der großen Mehrzahl der Flächenländer Standard. Nur drei Länder lassen den Kommunen die Wahlfreiheit zwischen Doppik und Kameralistik. Dass der Übergang zur Doppik Aufwand verursacht hat, ist nicht zu bestreiten; im Regelbetrieb nach mehreren Jahren spielt das aber keine Rolle mehr. Es mag sein, dass das doppische Regelwerk komplexer ist; aber den Sinn kalkulatorischer Zinsen oder die Bedeutung von Kasseneinnahmeresten in der Kameralistik haben auch damals viele nicht verstanden. Im Übrigen finden sich auch gestandene Kameralist:innen in der Doppik wieder. Denn in der Finanzrechnung können die für Investitionen veranschlagten Zahlungen wie bisher verfolgt werden.

In der Kameralistik war das Erstaunen groß, wenn nach einiger Zeit ein höherer Unterhaltungsaufwand erforderlich wurde. Denn der Titel „Bauliche Unterhaltung“ war ein gern genutzter Ansatzpunkt, um durch Reduzierung den formalen Haushaltsausgleich herzustellen. Zudem wurden größere Maßnahmen oft als Generalsanierung oder Ähnli-

(C) Der/die Autor:in 2021. Open Access: Dieser Artikel wird unter der Creative Commons Namensnennung 4.0 International Lizenz veröffentlicht (creativecommons.org/licenses/by/4.0/deed.de).

Open Access wird durch die ZBW - Leibniz-Informationszentrum Wirtschaft gefördert. ches bezeichnet, um damit den Spielraum für die Kreditfinanzierung zu erweitern. Wenn und soweit mit der Doppik eine schärfere Abgrenzung erfolgt, ist das sicher zu begrüßen. Das sollte Versuche, den Haushaltsausgleich durch Kürzung der regelmäßigen Bauunterhaltung rechnerisch anzustreben, zumindest erschweren.

Ein weiterer Aspekt in der Doppik ist die Vorsorge für künftige Verpflichtungen, so die Ruhestandsbezüge der Beamt:innen, die in der Kameralistik nicht zu erkennen waren. Die Zuführung zu den Rückstellungen soll zumindest ins Gedächtnis der Entscheidungstragenden rufen, dass da „noch etwas kommen kann". Da die Landesgesetzgebung allerdings hohe Abzinsungsfaktoren vorgesehen hat, ist die künftige Belastung mit Sicherheit noch zu gering angesetzt. Nicht vergessen darf man, dass den Zuführungen auch die Auflösung von Rückstellungen bei Inanspruchnahme gegenübersteht.

Der Kernpunkt der Doppik-Kritisierenden lautet: Der Haushaltsausgleich als Voraussetzung für die Investitionsfähigkeit ist schwieriger geworden. Wie sieht es nun mit der Belastung des Haushalts aus? Auf der Aufwandseite entstehen Mehraufwendungen durch die Abschreibungen, doch stehen ihnen Auflösungen aus Sonderposten für Zuschüsse und Beiträge gegenüber. Damit wird die Finanzierungsstruktur von Investitionen nicht nur einmalig, sondern bis zum Ende des Abschreibungszeitraums präsent.

Ein erhöhter Bearbeitungsaufwand entsteht in der Doppik unbestritten durch Inventarisierung und Bewertung. Dabei ist die Bewertung - abgesehen von der Erstbewertung - in den meisten Fällen nicht so kompliziert, da die Anschaffungs- und Herstellungskosten maßgeblich sind. Mehr Zeit erfordert die physische Inventur. Nur: Auch in der Kameralistik gab es die Verpflichtung, ein Inventarverzeichnis zu führen. Allerdings führte diese Bestimmung oft ein Schattendasein. Es mag sein, dass ein doppischer Haushalt komplexer ist. Der Umfang vieler Haushaltspläne könnte dies nahelegen. Aber die Nachwuchskräfte lernen längst die Doppik und können sich in dem System bewegen. Natürlich waren für die Doppik neue IT-Lösungen erforderlich, doch die Altverfahren der Kameralistik waren oft zumindest dringend überarbeitungsbedürftig.

Ist die Kameralistik trotzdem besser, weil in Thüringen nur $5 \%$ der kreisangehörigen Gemeinden doppisch buchen und in Bayern zwei Gemeinden zur Kameralistik zurückgekehrt sind? Soll die Kameralistik für kleinere Kommunen gelten? Warum aber soll die "investitionshemmende" Doppik dann in größeren Kommunen angebracht sein? Nein, ein Zurück kann und wird es nicht geben.

Gunnar Schwarting Deutsche Universität für Verwaltungswissenschaften Speyer g.schwarting@gmx.de 